\title{
Plasma Homocysteine Levels in Polycystic Ovary Syndrome and Congenital Adrenal Hyperplasia
}

\author{
FiRAT BAYRAKTAR, DidEM DERELI*, A. GOKHAN ÖZGEN* AND CANDEĞER YILMAZ* \\ Dokuz Eylul University Endocrinology Department, Balcova, 35350 Izmir, Turkey \\ *Ege University Endocrinology Department, Bornova, 35100 Izmir, Turkey
}

\begin{abstract}
The purpose of this study was to determine whether polycystic ovary syndrome (PCOS) and nonclassic 21hydroxylase deficiency $(\mathrm{CAH})$ are related to hyperhomocysteinemia, and to investigate if there is a correlation between homocysteine levels and insulin sensitivity in women with PCOS and CAH. Fifty patients with PCOS, 50 patients with $\mathrm{CAH}$ and 25 control women were included in the study. Blood samplings were performed in the early follicular phase for measuring hormone profile, Vitamin $\mathrm{B}_{12}$, folate, homocysteine levels and fasting blood glucose. Ovulatory status was assessed with timed serum progesterone measurements. Homeostasis model assessment-insulin resistance (HOMA-IR) was calculated as a measure of insulin resistance. Mean homocysteine levels were found as $(8.9+1.9 \mathrm{umol} / 1$ and $17.7+3.6 \mathrm{umol} / \mathrm{l})$ in the normal group and PCOS respectively $(\mathrm{p}<0.001)$, but there was no statistical significance between nonclassic 21-hydroxylase deficiency $(9.0+2.2 \mathrm{umol} / \mathrm{l})$ and control group. Most of the patients in PCOS group (35 of 50) were significantly insulin resistant. However, there was no insulin resistant patient in CAH or control group. When we compare the two subgroups of PCOS women, the patients with insulin resistance had significantly higher homocysteine levels than the ones who were not insulin resistant. There were positive correlations among serum homocysteine, insulin and androgen levels in PCOS patients. There were no correlations among these parameters in CAH and control groups. Increased homocysteine levels may contribute to increased cardiovascular disease risk in patients with PCOS. The reason for hyperhomocysteinemia seems to be related to insulin resistance but not high androgen levels.
\end{abstract}

Key words: Polycystic ovary syndrome, Homocysteine, Insulin resistance

(Endocrine Journal 51: 601-608, 2004)

POLYCYSTIC ovary syndrome (PCOS), a condition characterized by oligomenorrhea and androgen excess, is a common problem among women of reproductive age. Although the pathogenesis of the syndrome is still uncertain, there are many studies suggesting that PCOS may increase the risk for several conditions including insulin resistance, type 2 diabetes, dyslipidemia, hypertension, pregnancy-associated diabetes and hypertension, cardiovascular disease and some cancers [1]. Insulin resistance and resultant hyperinsulinemia are the most important clinical features of PCOS as it may contribute to long-term complications of the syndrome [2].

Risks for coronary heart disease have been reported

Received: February 6, 2003

Accepted: July 5, 2004

Correspondence to: Dr. Didem DERELI, Oyak sitesi 2/8 sokak No 2/13, Uckuyular/Izmir 35350 Turkey to be diabetes mellitus, insulin resistance, and hypertension; high serum levels of total cholesterol, LDL cholesterol, triglycerides and low levels of HDL cholesterol. Obesity and increased waist-to-hip ratio have likewise been reported to be independent risk factors for cardiovascular disease in women [3, 4]. Based on most of the risk factor profiles, women with PCOS would be expected to be at significantly increased risk for coronary heart disease $[5,6]$.

Hyperhomocysteinemia has now been shown to be an independent predictor of cardiovascular mortality in patients with known atherosclerosis [7]. In addition, some authors suggest a correlation between hyperhomocysteinemia and insulin resistance [8]. The aim of this study was to investigate the serum levels of homocysteine in women with polycystic ovary syndrome and the possible correlation between the homocysteine levels and insulin sensitivity. 


\section{Materials and Methods}

\section{Study population}

A total of 320 women with oligomenorrhea and androgen excess (clinical or biochemical) were evaluated. Among them, 50 women with the diagnosis of PCOS according to NICHD criteria [9] and 50 women with the diagnosis of nonclassic 21-hydroxylase deficiency according ACTH stimulation test [10] were selected. All age and BMI matched controls were examined to exclude patients with hyperandrogenic signs and symptoms (clinical-biochemical). The study was approved by the ethics committee and the Instutional Review Board of Ege University Medical Faculty and all women screened and included gave informed consent for the study.

The diagnosis of PCOS was based on the NICHD criteria: (i) hyperandrogenism and/or hyperandrogenemia, (ii) oligo-anovulation, (iii) exclusion of other known disorders, such as Cushing's syndrome, hyperprolactinemia or nonclassic congenital adrenal hyperplasia. Polycystic ovary appearance at ultrasonography was not considered a criterion for the diagnosis of the syndrome [9]. Hyperandrogenemia was defined as serum free testosterone $>3.2 \mathrm{pg} / \mathrm{ml}$; (normal range 0.8 $3.2 \mathrm{pg} / \mathrm{ml}$ ). Oligomenorrhea was defined as bleeding episode occurring less than six times per year. Anovulation was confirmed in all patients with serial weekly serum progesterone levels $(<2.5 \mathrm{ng} / \mathrm{mL}=<8.0 \mathrm{nmol} / \mathrm{L})$ starting on day 21 of their menstrual cycle.

Blood samples were obtained at 7:30-8:15 AM during the early follicular phase (1st-5th days) after spontaneous or in progesterone-induced menses in women. Medroxyprogesterone acetate (Farlutal, Deva, Istanbul; $10 \mathrm{mg} / \mathrm{D}$ for 10 days) was prescribed to induce progesterone withdrawal bleeding, when necessary. The serum samples were stored at $-20^{\circ} \mathrm{C}$ until assayed.

Early follicular phase serum $17-\mathrm{OH}$ progesterone levels were measured in the morning to avoid later elevations, levels $>1.5 \mathrm{ng} / \mathrm{mL}$ were considered as suspected levels for late onset CAH. For the diagnosis of $\mathrm{CAH}$, serum 17-OHP levels were measured before, 30 and 60 minutes after injection of $0.25 \mathrm{mg}$ synthetic ACTH (1-24) (Synacthen, Ciba, Switzerland) given IV at 8:00 AM on early follicular phase. An ACTHstimulated 17-OHP levels $>10 \mathrm{ng} / \mathrm{mL}$ was considered the criterion of late-onset 21-hydroxylase deficiency.

Thyroid dysfunction, hyperprolactinemia and hyper- cortisolism were all excluded using appropriate tests. Patients had not received oral contraceptives or any medications that are known to alter hormone, lipid or insulin metabolism within 3 months before the study.

\section{Ultrasonographic examinations}

Pelvic ultrasound examinations were performed by using a $3.5 \mathrm{MHZ}$ abdominal transducer (98 women) and a $6.5 \mathrm{MHz}$ vaginal end probe (27 women) (EUB 450; Hitachi, Tokyo, Japan). All sonograms were obtained early in the cycle. In PCOS group, 36 women had polycystic ovaries, 10 had multifollicular ovaries and, in the remaining four patients, the ovaries were sonographically of normal appearance. In nonclassic 21-hydroxylase deficiency group, 22 women had polycystic ovaries, 15 had multifollicular ovaries and the remaining 13 patients' ovaries had normal appearance on sonography. In control group, there were no sonographic abnormal appearances (abnormality in ovarian USG was an exclusion criteria for the control group).

\section{Assay methods}

The serum concentrations of $\mathrm{FSH}, \mathrm{LH}, \mathrm{E}_{2}$, progesterone, prolactin, and cortisol, were measured by chemiluminescent enzyme immunoassay (ASC $180(+)$ Ciba Diagnostics, USA) with average interassay coefficient variation $(\mathrm{CV})$ of $8 \%$ and intra-assay $\mathrm{CV}$ of $7 \%$. The serum concentrations of DHEA-S, $17-\mathrm{OH}$ progesterone, Free Testosterone, Free $\mathrm{T}_{4}$ and TSH were measured with standard radioimmunoassay.

Blood samples for hormonal tests were collected after a 16-hour fasting, immediately placed on ice, and centrifuged at $3,500 \mathrm{~g}$ for $30 \mathrm{~min}$ at $+4^{\circ} \mathrm{C}$. Plasma was separated within $1 \mathrm{~h}$ and stored at $-70^{\circ} \mathrm{C}$.

Plasma glucose was measured by glucose oxidase technique (Biobak Laboratory Supplies Trade, Ankara, Turkey) with an interassay CV of $1.7 \%$ and intra-assay $\mathrm{CV}$ of $0,8 \%$. Insulin levels were measured by microparticle enzyme immunoassay (Abbott, WiesbadenDelkenheim, Germany) with interassay and intraassay CVs $2.4 \%$.

Serum concentrations of vitamin $\mathrm{B}_{12}$ and folate were measured by chemiluminescent enzyme immunoassay (Immulite 2000 Diagnostic Products Corporation, Los Angeles, CA) with average interassay and intraassay CVs for vitamin $\mathrm{B}_{12}$ of $7.4 \%$ and $6.5 \%$ respectively. These figures were $4.0 \%$ and $4.2 \%$ for folate measure- 
ments.

Serum high-density lipoprotein (HDL), total cholesterol, and triglyceride concentrations were measured by enzymatic assay (Boehringer-Mannheim, Germany). Serum lipoprotein (a) levels were measured by a solidphase, two-site immunoradiometric assay (Pharmacia, Uppsala, Sweden), with average interassay CV of $6.6 \%$ and intra-assay $\mathrm{CV}$ of $5.0 \%$. The low-density lipoprotein (LDL) level was calculated using the Friedewald equation:

$\mathrm{LDL}=$ total cholesterol $-\mathrm{HDL}-($ triglyceride/5)

Serum concentrations of homocysteine were measured by high performance liquid chromatography with the Hewlett-Packard 1100 Series System (Waldron, Germany).

HOMA-IR was calculated according to the following formula:

HOMA-IR $=$ FIRI $\times$ FPG $/ 22.5$ [FIRI: fasting plasma insulin level $(\mu \mathrm{U} / \mathrm{ml})$ and FPG is fasting plasma glucose level (nmol/L)] [11]

Body mass index (BMI) was calculated according to the following formula:

$\mathrm{BMI}=$ body weight $(\mathrm{kg}) /$ body height squared (meter)

\section{Statistical analysis}

The Statistical Package for the Social Sciences (SPSS version 10.0 for Windows) was used for statistical analyses. The characteristics of distribution were tested with the Kolmogorov-Smirnov test. Results were expressed as mean $\pm \mathrm{SD}$. Differences between means were analyzed by Student's unpaired $t$-test using two tailed tests for significance. Because of differing numbers of subjects in each group, the Tukey-Kramer multiple comparison procedure was used for post hoc comparisons. Correlations between homocysteine and insulin resistance were evaluated again after excluding BMI with covariance analysis because BMI might affect insulin sensitivity. $P$ values smaller than 0.05 was regarded as statistically significant.

\section{Results}

The mean demographic, endocrinological and metabolic characteristics of the groups are given in Table 1. Age, BMI and waist-to-hip ratios did not differ significantly between the patients and the controls. The mean serum FSH, prolactin, estradiol levels were compara-

Table 1. Clinical data and hormonal findings in patients with PCOS, CAH and healthy women

\begin{tabular}{|c|c|c|c|c|}
\hline VARIABLE & $\operatorname{PCOS}(n=50)$ & $\mathrm{CAH}(\mathrm{n}=50)$ & CONTROL $(\mathrm{n}=25)$ & $P$ value \\
\hline AGE (years) & $21.4 \pm 3.16$ & $22.1 \pm 2.91$ & $21.6 \pm 3.18$ & NS \\
\hline BMI $\left(\mathrm{kg} / \mathrm{m}^{2}\right)$ & $23.9 \pm 4.32$ & $22.05 \pm 3.21$ & $23.4 \pm 2.55$ & NS \\
\hline Waist/Hip & $0.79 \pm 0.06$ & $0.77 \pm 0.05$ & $0.78 \pm 0.03$ & NS \\
\hline FSH (IU/L) & $4.78 \pm 1.66$ & $5.31 \pm 1.36$ & $5.44 \pm 1.99$ & NS \\
\hline LH (IU/L) & $11.9 \pm 7.05$ & $6.54 \pm 3.61$ & $6.11 \pm 2.98$ & $\mathrm{P}<0.01^{\uparrow *} ; \mathrm{NS}^{\S}$ \\
\hline Prolactin $(\mathrm{ng} / \mathrm{mL})$ & $13.6 \pm 11.4$ & $15.3 \pm 10.4$ & $14.4 \pm 12.3$ & NS \\
\hline DHEA-S $(\mu \mathrm{g} / \mathrm{dL})$ & $178.1 \pm 121.3$ & $261.4 \pm 117.4$ & $177.2 \pm 99.7$ & $\mathrm{P}<0.05$ ॠ\$; NS* \\
\hline F.Testo (pg/mL) & $3.01 \pm 1.67$ & $3.12 \pm 1.33$ & $1.67 \pm 2.01$ & $\mathrm{P}<0.01 * \S ; \mathrm{NS}$ \\
\hline Estradiol (pg/mL) & $45.6 \pm 25.4$ & $43.7 \pm 29.7$ & $41.9 \pm 13.2$ & NS \\
\hline Glucose (mg/dL) & $94.3 \pm 8.4$ & $88.2 \pm 5.44$ & $89.4 \pm 5.38$ & $\mathrm{P}<0.05^{\top *} ; \mathrm{NS}^{\S}$ \\
\hline Insulin $(\mu \mathrm{IU} / \mathrm{mL})$ & $17.2 \pm 6.5$ & $10.4 \pm 3.1$ & $9.9 \pm 3.2$ & $\mathrm{P}<0.01^{\uparrow *} ; \mathrm{NS}^{\S}$ \\
\hline HOMA-IR & $3.49 \pm 0.17$ & $2.18 \pm 0.11$ & $2.16 \pm 0.09$ & $\mathrm{P}<0.001^{\top} * ; \mathrm{NS}^{\S}$ \\
\hline T. Chol. (mg/dL) & $182.7 \pm 25.7$ & $177.1 \pm 31.9$ & $181.4 \pm 29.6$ & NS \\
\hline Triglyceride $(\mathrm{mg} / \mathrm{dL})$ & $137.7 \pm 97.9$ & $100.7 \pm 11.8$ & $89.6 \pm 10.7$ & $\mathrm{P}<0.01^{\uparrow *} ; \mathrm{NS}^{\S}$ \\
\hline $\mathrm{LDL}(\mathrm{mg} / \mathrm{dL})$ & $103.8 \pm 23.8$ & $98.9 \pm 21.7$ & $99.6 \pm 22.4$ & NS \\
\hline HDL (mg/dL) & $44.4 \pm 11.9$ & $57.9 \pm 10.7$ & $59.8 \pm 9.9$ & $\mathrm{P}<0.01^{\text {I*}} ; \mathrm{NS}^{\S}$ \\
\hline Lipopro. (a) (mg/dL) & $25.7 \pm 18.7$ & $22.7 \pm 19.6$ & $23.4 \pm 13.8$ & NS \\
\hline Homocysteine $(\mu \mathrm{mol} / \mathrm{L})$ & $17.7 \pm 3.6$ & $9.0 \pm 2.2$ & $8.9 \pm 1.9$ & $\mathrm{P}<0.001^{\top *} ; \mathrm{NS}^{\S}$ \\
\hline Vitamin $B_{12}(\mathrm{pg} / \mathrm{mL})$ & $251.9 \pm 122.3$ & $267.8 \pm 132.2$ & $156.2 \pm 129.8$ & NS \\
\hline Folate $(\mathrm{ng} / \mathrm{dL})$ & $9.3 \pm 3.1$ & $9.2 \pm 2.9$ & $9.3 \pm 2.7$ & NS \\
\hline
\end{tabular}

NOTE: All values are means \pm SD. NS: not significant, BMI: body mass index, F. Testo: free testosterone, $\uparrow$ : PCOS-CAH- §: CAH-Control- *: PCOS-Control 
Table 2. Clinical data and hormonal findings in patients with PCOS; with or without insulin resistance

\begin{tabular}{lccc}
\hline \multicolumn{1}{c}{ VARIABLE } & PCOS-IR $(\mathrm{n}=35)$ & PCOS-IS $(\mathrm{n}=15)$ & $P$-Value \\
\hline AGE $($ years $)$ & $20.9 \pm 2.31$ & $22.5 \pm 3.47$ & $\mathrm{NS}$ \\
BMI $\left(\mathrm{kg} / \mathrm{m}^{2}\right)$ & $24.9 \pm 3.21$ & $23.6 \pm 4.02$ & $\mathrm{NS}$ \\
Waist/Hip & $0.80 \pm 0.10$ & $0.78 \pm 0.02$ & $\mathrm{NS}$ \\
FSH $(\mathrm{IU} / \mathrm{L})$ & $4.68 \pm 1.33$ & $4.84 \pm 1.93$ & $\mathrm{NS}$ \\
LH $(\mathrm{IU} / \mathrm{L})$ & $7.79 \pm 6.17$ & $12.9 \pm 8.25$ & $\mathrm{P}<0.01$ \\
Prolactin $(\mathrm{ng} / \mathrm{mL})$ & $12.7 \pm 11.3$ & $13.9 \pm 12.4$ & $\mathrm{NS}$ \\
DHEA-S $(\mu \mathrm{g} / \mathrm{dL})$ & $182.1 \pm 131.3$ & $173.1 \pm 133.6$ & $\mathrm{NS}$ \\
F.Testo $(\mathrm{pg} / \mathrm{mL})$ & $3.41 \pm 1.58$ & $2.83 \pm 1.04$ & $\mathrm{P}<0.01$ \\
Estradiol $(\mathrm{pg} / \mathrm{mL})$ & $46.8 \pm 23.4$ & $44.3 \pm 27.1$ & $\mathrm{NS}$ \\
Glucose $(\mathrm{mg} / \mathrm{dL})$ & $99.7 \pm 11.5$ & $87.1 \pm 4.4$ & $\mathrm{P}<0.01$ \\
Insulin $(\mu \mathrm{IU} / \mathrm{mL})$ & $19.6 \pm 8.5$ & $11.2 \pm 4.1$ & $\mathrm{P}<0.01$ \\
HOMA-IR & $4.78 \pm 1.25$ & $2.39 \pm 0.21$ & $\mathrm{P}<0.001$ \\
T. Chol. $(\mathrm{mg} / \mathrm{dL})$ & $182.7 \pm 25.7$ & $182.7 \pm 25.7$ & $\mathrm{NS}$ \\
Triglyceride $(\mathrm{mg} / \mathrm{dL})$ & $142.7 \pm 69.9$ & $128.3 \pm 67.5$ & $\mathrm{P}<0.05$ \\
LDL $(\mathrm{mg} / \mathrm{dL})$ & $104.6 \pm 19.8$ & $102.8 \pm 27.2$ & $\mathrm{NS}$ \\
HDL $(\mathrm{mg} / \mathrm{dL})$ & $41.3 \pm 14.9$ & $48.4 \pm 9.6$ & $\mathrm{P}<0.05$ \\
Lipopro. $(\mathrm{a})(\mathrm{mg} / \mathrm{dL})$ & $25.5 \pm 19.6$ & $24.9 \pm 19.6$ & $\mathrm{NS}$ \\
Homocysteine $(\mu \mathrm{mol} / \mathrm{L})$ & $19.8 \pm 5.8$ & $14.5 \pm 2.9$ & $\mathrm{P}<0.01$ \\
Vitamin $\mathrm{B}_{12}(\mathrm{pg} / \mathrm{mL})$ & $249.6 \pm 117.5$ & $252.7 \pm 147.9$ & $\mathrm{NS}$ \\
Folate $(\mathrm{ng} / \mathrm{dL})$ & $9.4 \pm 3.2$ & $9.3 \pm 4.1$ & $\mathrm{NS}$ \\
\hline
\end{tabular}

NOTE: All values are means \pm SD. NS: not significant, BMI: body mass index PCOS-IR: polycystic ovary syndrome with insulin resistance

PCOS-IS: polycystic ovary syndrome without insulin resistance

ble among the three groups. The LH levels in PCOS group were significantly higher than the other two groups' LH levels $(\mathrm{p}<0.01)$; serum DHEAS levels in patients with $\mathrm{CAH}$ were significantly higher than the other two groups' DHEAS levels $(\mathrm{p}<0.01)$. Serum free testosterone (FT) levels were significantly higher in both patients with PCOS and CAH compared with the control group $(\mathrm{p}<0.01)$. There was no significant difference between PCOS and CAH groups regarding estradiol values ( $p>0.05)$.

Serum mean fasting glucose and insulin levels from patients with PCOS were significantly higher than the other groups $(p<0.05$ and $p<0.01$, respectively). The HOMA-IR was significantly higher in PCOS group indicated an insulin resistance in these patients.

The mean serum levels of cholesterol and LDL were comparable among the groups. However, the mean triglyceride concentration was significantly higher and the mean HDL concentration was significantly lower in PCOS group than the other two groups $(\mathrm{p}<0.01)$.

The mean serum homocysteine concentration was significantly higher in PCOS group than the other groups $(17.7 \pm 3.6$ versus $9.0 \pm 2.2$ and $8.9 \pm 1.9 \mathrm{p}<0,001)$. There was no significant difference between homocys- teine levels of CAH group and control group ( $>>0.05)$.

The mean serum concentrations of vitamin $B_{12}$ and folate were comparable among the three groups $(\mathrm{P}>0.05)$.

Correlation analyses were performed between the homocysteine levels and various metabolic and hormonal parameters in PCOS group. There was a strong positive correlation between the mean homocysteine levels and mean fasting insulin levels in PCOS patients $(\mathrm{r}=0.68 ; \mathrm{p}<0.01)$. The mean homocysteine levels also correlated positively with the HOMA-IR $(r=0.69$; $\mathrm{p}<0.01)$. There was a slight positive correlation between free testosterone and homocysteine levels of PCOS patients $(r=0.67 ; p<0.05)$. But when we rule out the effect of insulin with covariant analysis the correlation was not significantly significant. The correlation of homocysteine and free testosterone levels in CAH group was not statistically significant $(r=0.60$; $\mathrm{p}>0.05)$.

\section{Insulin resistance in PCOS group}

Various results of HOMA-IR were observed in women with PCOS. When we take 2.7 for the cut off value 
for insulin resistance, 35 women with PCOS had insulin resistance while the remaining 15 did not.

We therefore divided the patients with PCOS into two groups according to their insulin sensitivity. Table 2 shows the main characteristics of the two PCOS patient groups.

Age, BMI and waist-to-hip ratios did not differ significantly between the two PCOS patient groups. The mean serum FSH, prolactin, estradiol levels were also comparable between the two groups $(p>0.05)$. The mean LH level of insulin sensitive group was significantly higher then the insulin resistant group $(p<0.01)$. The mean serum free testosterone level of insulin resistant group was significantly higher than the insulin sensitive group $(p<0.01)$. Mean serum triglyceride levels were significantly higher and HDL levels were significantly lower in insulin resistant PCOS patients $(\mathrm{p}<0.05)$.

When we compare the mean homocysteine levels of the two PCOS patient subgroups, although homocysteine levels from insulin sensitive group were higher than the control group $(p<0.05)$, their levels were lower than insulin resistant PCOS patients $(p<0.01)$.

Correlation analyses were performed again between the homocysteine levels and the significant parameters in insulin resistant PCOS group. There was a positive correlation between the mean homocysteine level and mean fasting insulin level in insulin resistant patients $(\mathrm{r}=0.89 ; \mathrm{p}<0.01)$. The mean homocysteine levels also correlated positively with the HOMA-IR $(r=0.84$; $\mathrm{p}<0.001)$.

\section{Discussion}

Polycystic ovary syndrome (PCOS) is an exceptionally common disorder of premenopausal women characterized by hyperandrogenism and chronic anovulation with the exclusion of specific disorders, such as nonclassic adrenal 21-hydroxylase deficiency, hyperprolactinemia, or androgen secreting neoplasm [9]. The polycystic ovary morphology is consistent with, but not essential for, the diagnosis of the syndrome.

Although its etiology remains unknown, the association between insulin resistance and compensatory hyperinsulinemia has added new insights into the pathogenesis of the syndrome [12]. Women with PCOS are now accepted to have increased risk of developing diabetes mellitus type 2, dyslipidemia, hypertension, and atherosclerosis [13].

Hyperhomocysteinemia is a cardiovascular risk factor independent of other classic risk factors. The exact mechanism concerning the manner in which hyperhomocysteinemia causes vascular damage has not been clarified completely [7]. It has been proposed that it directly damages endothelial cells, disrupts platelet function, and generates formation of reactive oxygen species, which in turn cause auto oxidation [14].

There are only a few preliminary studies that have explored mean homocysteine levels in women with PCOS and the significance of the relationship between PCOS and hyperhomocysteinemia is still controversial [15-17].

In the present study, we reported an increase in plasma homocysteine levels above normal limits in PCOS patients, and we demonstrated that there is a relationship between plasma homocysteine levels and insulin resistance. We also measured plasma homocysteine levels in women with NC-CAH to determine the effects of serum androgen to plasma homocysteine levels, as women with $\mathrm{NC}-\mathrm{CAH}$ have nearly the same clinical and hormonal evidence of hyperandrogenemia without insulin resistance.

The first report about hyperhomocysteinemia in women with PCOS is by Yarali et al. [15]. They demonstrated hyperhomocysteinemia and insulin resistance in women with PCOS but they failed to demonstrate a significant correlation between insulin sensitivity and homocysteine levels. Although they did not mention any significant correlation among insulin, uric acid, and homocysteine levels, they have demonstrated an elevation in serum uric acid concentration, which might have an indirect effect on serum homocysteine levels. They also did not investigate the relationship between serum androgen and homocysteine levels but there are indications that plasma homocysteine levels are influenced by sex steroid hormones [18]. We demonstrated higher homocysteine levels than previous American studies in all our study groups. Yarali et al. demonstrated the same elevated homocysteine levels even in the control subjects. The higher homocysteine levels in Turkish population may be due to different ethnicity. Similar ethnic difference was also demonstrated in HDL levels of Turkish population [19].

Still et al. were not able to find any statistically significant difference in plasma homocysteine levels between women with normal and those with polycystic ovaries [20]. The discrepancy may be due to differ- 
ences in the criteria used to diagnose PCOS, as they did not use the NICHD conference consensus criteria [21]. In our opinion, they have evaluated a different patient group than the other studies about homocysteine levels in patients with PCOS.

Vrbikova et al. demonstrated an increase in the serum homocysteine levels after long-term metformin treatment in women with PCOS [17]. Basal homocysteine levels in their study group were within upper normal limits after almost 6 months of metformin $1000 \mathrm{mg}$ /day therapy. They failed to show any correction in insulin sensitivity and almost no androgen hormone level change was observed during their study. This may be in part because of the relatively low medication dose. In addition, they did not measure serum folate and vitamin $B_{12}$ levels in the beginning and at the end of the study. Metformin decreases these vitamin levels by inhibiting their absorption from the intestinal system. Moreover, folate and vitamin $\mathrm{B}_{12}$ deficiencies are known to cause a significant elevation in plasma homocysteine levels [22].

Although the mean homocysteine levels in their study groups were within normal limits, Loverro et al. have found significantly higher mean plasma homocysteine concentrations in patients with PCOS as compared with controls [16]. However, they failed to demonstrate a correlation between insulin sensitivity and homocysteine levels. Moreover, they did not mention any relation between androgen excess and hyperhomocysteinemia. Therefore, they failed to explain the mechanism underlying hyperhomocysteinemia. They suggested further studies for evaluating serum homocysteine levels and the mechanism that might underlie the possible hyperhomocysteinemia in women with PCOS [16].

In the present study, we reported increased plasma homocysteine levels in young lean women affected by PCOS than age and BMI matched patients with NC$\mathrm{CAH}$ and controls. We chose the patients with NC$\mathrm{CAH}$ because circulating testosterone, androstenedione, and dehydroepiandrostenedione sulfate levels are not different from patients with ovarian hyperandrogenism [10]. In addition, the mechanism underlying androgen excess in NC-CAH is an enzyme defect that is not insulin resistant [10].

Deficiencies in cofactors folate and $\mathrm{B}_{12}$ have been demonstrated to elevate plasma homocysteine [7]. These parameters were comparable among the three groups so we cannot explain the homocysteine eleva- tion with cofactor deficiency in PCOS women. The renal functions of all three group patients were normal and so renal insufficiency is unlikely to be the reason for hyperhomocysteinemia in women with PCOS. Homocysteine is an independent risk factor and is not dependent of lipid metabolism [7]. We did not demonstrate any correlation between any lipid parameters and homocysteine levels when we excluded the effect of insulin in this correlation with covariant analysis.

Although Tsanadis et al. reported that the odds ratio for bearing a mutation on the methylene tetrahydrofolate reductase gene was 1.2-fold higher in women with PCOS than those without it, the mild elevation of the homocysteine levels in our PCOS patients are lower then expected as those of congenital enzymatic deficient patients could be [23].

Although the significant relationship between insulin levels and hyperhomocysteinemia is still debated, there are growing lines of evidence that link hyperhomocysteinemia to diabetes or insulin resistance. For example, Audelin and Genest have demonstrated type 2 diabetic patients with cardiovascular disease have elevated plasma homocysteine levels. This elevation was not observed in type 1 diabetic subjects [24]. Dicker-Brown et al. have demonstrated an inhibitory effect of hyperinsulinemia on the expression and activity of tetrahydrofolat reductase and cystathionine synthase in rat hepatocytes [25]. Gursu et al. also demonstrated a dose-dependent manner increase of homocysteine level by insulin administration. Insulin appears to regulate plasma homocysteine levels by decreasing the transsulfuration (cystathione synthases are enzymes that catalyze the transsulfuration reactions) activity of enzymes in hepatocytes [26].

The inverse relationship between insulin sensitivity and plasma homocysteine levels that we have demonstrated in our study is a further support for this hypothesis. In our PCOS women population, 35 women (70\%) were insulin resistant.

For further investigation of the effect of insulin resistance on serum homocysteine levels, we divided our PCOS women group into two subgroups due to their insulin sensitivity. The ones who had HOMA-IR level above 2.7 were considered as insulin resistant and were placed in subgroup 1; the other women whose HOMAIR were lower than 2.7 are considered as sensitive to the actions of insulin and were placed in subgroup 2 . The mean serum homocysteine level of subgroup 1 was significantly higher than mean homocysteine levels 
of the subgroup 2. The correlation between serum homocysteine levels and serum insulin sensitivity was stronger in insulin resistant subgroup.

This observation provides interesting evidence for the effect of insulin sensitivity on serum homocysteine levels. The patients that we have accepted as insulin sensitive had lower HOMA-IR levels than the cut-off value for insulin resistance. However, they had significantly higher HOMA-IR levels as compared to $\mathrm{NC}-\mathrm{CAH}$ and control subjects, and so we cannot rule out the effect of insulin on homocysteine levels.

We concluded that serum homocysteine levels, an independent risk factor for coronary artery disease, are elevated in patients with PCOS, and that this elevation is associated more with insulin sensitivity then androgen excess. Further investigations are needed to confirm the role of insulin sensitivity on plasma homocysteine levels and the effect of the insulin-sensitizing drugs on this elevation.

\section{References}

1. Solomon CG (1999) The epidemiology of polycystic ovary syndrome. Prevalence and associated disease risks. Endocrinol Metab Clin North Am 28: 247-263.

2. Auernhammer CJ, Engelhardt D, Parhofer KG (2002) Polycystic ovary syndrome and metabolic syndrome. Dtsch Med Wochenschr 3, 127: 971-977.

3. Wild S, Pierpoint T, Jacobs H, McKeigue P (2000) Long-term consequences of polycystic ovary syndrome: results of a 31 years follow-up study. Hum Fertil (Camb) 3: 101-105.

4. Knopp RH (2002) Risk factors for coronary artery disease in women. Am J Cardiol 20: 89 (12 Suppl): 28E-34E; discussion 34E-35E.

5. Stein EA (2002) Identification and treatment of individuals at high risk of coronary heart disease. $\mathrm{Am} \mathrm{J}$ Med 3; 112 Suppl 8A: 3S-9S.

6. Mather KJ, Kwan F, Corenblum B (2000) Hyperinsulinemia in polycystic ovary syndrome correlates with increased cardiovascular risk independent of obesity. Fertil Steril 73: 150-156.

7. Nehler MR, Taylor LM Jr, Porter JM (1997) Homocysteinemia as a risk factor for atherosclerosis: a review. Cardiovasc Surg Dec 5: 559-567.

8. Giltay EJ, Hoogeveen EK, Elbers JM, Gooren LJ, Asscheman H, Stehouwer CD (1998) Insulin resistance is associated with elevated plasma total homocysteine levels in healthy, non-obese subjects [Letter]. Atherosclerosis 139: 197-198.

9. Zawadzki JK, Dunaif A (1992) Diagnostic criteria for polycystic ovary syndrome: towards a national approach. In: Dunaif A, Givens JR, Hasetline F, Merriam GR (eds) Polycystic Ovary syndrome. Boston: Blackwell; 377-384.

10. Azziz R, Dewailly D, Owerbach D (1994) Clinical review 56: Nonclassic adrenal hyperplasia: current concepts. J Clin Endocrinol Metab 78: 810-815.

11. Matthews DR, Hosker JP, Rudenski A, Turner RC (1985) Homeostasis model assessment: insulin resistance and beta cell function in man. Diabetologia 28:
401-411.

12. Dunaif A (1997) Insulin resistance and polycystic ovary syndrome: Mechanism and implications for pathogenesis. Endocr Rev 18: 774-800.

13. Ovalle F, Azziz R (2002) Insulin resistance, polycystic ovary syndrome, and type 2 diabetes mellitus. Fertil Steril 77: 1095-1105.

14. Munshi MN, Stone A, Fink L, Fonseca V (1997) Oxidative stress in diabetic macrovascular disease: does homocysteine play a role? South Med J 90: 903-906.

15. Yaralı H, Yıldırır A, Aybar F, Kabakçı G, Bukulmez O, Akgul E, Oto A (2001) Diastolic dysfunction and increased serum homocysteine concentrations may contribute to increased cardiovascular risk in patients with polycystic ovary syndrome. Fertil Steril 76: 511-516.

16. Loverro G, Lorusso F, Mei L, Depalo R, Cormio G, Selvaggi L (2002) The plasma homocysteine levels are increased in polycystic ovary syndrome. Gynecol Obstet Invest 53: 157-162.

17. Vrbikova J, Bicikova M, Tallova J, Hill M, Starka L (2002) Homocysteine and steroids levels in metformin treated women with polycystic ovary syndrome. Exp Clin Endocrinol Diabetes 110: 74-76.

18. Giltay EJ, Hoogeveen EK, Elbers JM, Gooren LJ, Asscheman H, Stehouwer CD (1998) Effects of sex steroids on plasma total homocysteine levels: a study in transsexual males and females. J Clin Endocrinol Metab 83: 550-553.

19. Onat A (2001) Risk factors and cardiovascular disease in Turkey. Atherosclerosis 156: 1-10.

20. Still ES, Genton MG, Perloe M, Schattman GL, Bralley JA, Tucker MJ (2001) Plasma homocysteine, fasting insulin, and androgen patterns among women with polycystic ovaries and infertility. Obstet Gynaecol Res 27: 163-168.

21. Gonzalez F, Chang L, Horab T, Stanczyk F, Crickard K, Lobo R (1999) Adrenal dynamic responses to physiologic and pharmacologic adrenocorticotropic hormone stimulation before and after ovarian steroid modulation 
in women with polycystic ovary syndrome. Fertil Steril 71: 439-444.

22. Desouza C, Keebler M, McNamara DB, Fonseca V (2002) Drugs affecting homocysteine metabolism: impact on cardiovascular risk. Drugs 62: 605-616.

23. Tsanadis G, Vartholomatos G, Korkontzelos I, Avgoustatos F, Kakosimos G, Sotiriadis A, Tatsioni A, Eleftheriou A, Lolis D (2002) Polycystic ovarian syndrome and thrombophilia. Hum Reprod 17: 314319.

24. Audelin MC, Genest J Jr (2001) Homocysteine and cardiovascular disease in diabetes mellitus. Atherosclerosis 159: 497-511.
25. Dicker-Brown A, Fonseca VA, Fink LM, Kern PA (2001) The effect of glucose and insulin on the activity of methylene tetrahydrofolat reductase and cystathionebeta-synthase: studies in hepatocytes. Atherosclerosis 158: 297-301.

26. Gursu MF, Baydas G, Cikim G, Canatan H (2002) Insulin increases homocysteine levels in a dosedependent manner in diabetic rats. Arch Med Res 33: 305-307.

27. Carmina E, Lobo RA (1999) Polycystic ovary syndrome (PCOS): arguably the most common endocrinopathy is associated with significant morbidity in women. J Clin Endocrinol Metab 84: 1897-1999. 